In-vivo real-time control of gene expression: a comparative analysis of feedback control

\title{
strategies in yeast
}

Gianfranco Fiore, ${ }^{\dagger, t, \S}$ Giansimone Perrino, ${ }^{\dagger, t, \S}$ Mario di Bernardo, ${ }^{*, t,}$ and Diego di Bernardo*,t,

TeleThon Institute of Genetics and Medicine (TIGEM), Pozzuoli, Italy, Department of Electrical Engineering and Information Technology, University of Naples Federico II, Naples, Italy, and Department of Engineering Mathematics, University of Bristol, Bristol, $U K$

E-mail: mario.dibernardo@unina.it; dibernardo@tigem.it

- Supporting Information Appendix -

\section{GAL1 promoter mathematical model derivation}

The MPC and the ZAD control strategies need a mathematical model of the process being controlled to calculate the control input $u$. Hence to implement these regulators we have derived a mathematical representation for the transcription driven by the GAL1 promoter.

\footnotetext{
*To whom correspondence should be addressed

†TIGEM

$\ddagger$ University of Naples Federico II

"University of Bristol

$\S$ These authors contributed equally to this work
} 
The same model has been used to tune the PI controller gains and as a proxy to perform in-silico control experiments.

\section{Data set}

We have produced two different experimental data set:

1. Data set 1: yeasts have been imaged for up to 16 hours. During this interval Galactose and Glucose have been alternatively provided to the yeast chamber. Cells were fed with a Galactose enriched medium for 180 min and then with Glucose for the following $180 \mathrm{~min}$ and so on until the end of the experiment, as depicted in Supporting Figure 1, Lower Panel. The average Gfp fluorescence of the cell population, in the green spectrum, has been instead taken as the system output (Supporting Figure 1, Upper Panel).

2. Data set 2: we have performed a longer experiment (2180 minutes), in which after keeping cells for the first 180 minutes in Galactose, a random sequence of pulses in between Galactose and Glucose has been provided to cells to elicit GAL1 promoter activity. For model identification, we have considered the switches in between Galactose and Glucose as the input (Supporting Figure 2, Lower Panel) and the measure of the average fluorescence intensity expressed by the cells as the output (Supporting Figure 2, Upper Panel).

\section{Candidate model, identification strategy and validation methodology}

According to the results obtained and described in (1), we decided to fit, on the experimental data, the parameters of the following two dimensional state-space linear model defined as : 


$$
\begin{aligned}
& \frac{d x_{1}}{d t}=-d_{1} x_{1}+b u \\
& \frac{d x_{2}}{d t}=v_{2} x_{1}-d_{2} x_{2}
\end{aligned}
$$

In Equation (1), $u$ is the only external input to the model and it is assumed to be equal to 2 when cells are fed with Galactose, whereas, when Glucose is provided to yeasts, it is assumed to be equal to 0 (these values are related to the concentration of Galactose in the growing medium). The estimated parameters are: $d_{1}$ and $b$ in $(1), d_{2}$ and $v_{2}$ in (2) together with the initial conditions.

Four different scenarios were considered for the estimation and the validation of the parameters of Equations (1) and (2):

\section{- Scenario I}

The parameters of the model were estimated on the data set 1 depicted in Supporting Figure 1. The same data set was used for the validation.

\section{- Scenario II}

The parameters of of the model were estimated on the data set 1 depicted in Supporting Figure 1. The cross-validation was completed on the data set 2 depicted in Supporting Figure 2.

\section{- Scenario III}

The parameters of the model were estimated on the data set 2 depicted in Supporting Figure 2. The same data were used for the validation process.

\section{- Scenario IV}

The parameters of of the model were estimated on the data set 2 depicted in Supporting

Figure 2. The cross-validation was completed on the data set 1 depicted in Supporting Figure 1. 
Thus at the end of the identification process we obtained two different linear models inferred using experimental data represented in Supporting Figures 1 (Scenarios I and II) and 2 (Scenarios III and IV); the parameters, and the initial conditions $x_{1}(0), x_{2}(0)$, were estimated using the Prediction Error Minimisation (PEM) method (2). The models' performance in reproducing the data used for the identification and the other data set available (cross-validation), were assessed by calculating the indices described in the next paragraph.

\section{Metrics and Validation}

To assess the performance of each of the models and to carry out a comparison among the different scenarios, we have used the following metrics:

\section{Akaike's Final Prediction Error and Information Criterion}

Akaike's Final Prediction Error (FPE) or Aikake's Information Criterion (AIC) (2) can be used to evaluate the quality of a given model by testing how it captures the system response to a known input signal. The metrics can be computed as follows:

$$
\mathrm{FPE}:=\left(\frac{1+\frac{m}{N}}{1-\frac{m}{N}}\right) \frac{1}{N} \sum_{i=1}^{N} \epsilon^{2}\left(i, \theta_{N}\right),
$$

and

$$
\mathrm{AIC}:=\log \left(\frac{1}{N} \sum_{i=1}^{N} \epsilon^{2}\left(i, \theta_{N}\right)\right)+\frac{m}{N},
$$

where $\theta_{N}$ is the vector of estimated parameters, $m$ is the number of estimated parameters, $N$ is the dimension of the estimation dataset and $\epsilon\left(i, \theta_{N}\right)$ are the prediction errors. Both FPE and AIC take their smallest values when the model is the most accurate.

\section{Fitting percentage}

This index provides a measure of the percentage of the output variation that is reproduced by the model and is given by the following formula (2): 


$$
\operatorname{FIT}:=100\left(1-\frac{\sqrt{\sum_{i=1}^{N}\left(\widehat{y}_{i}-y_{i}\right)^{2}}}{\sqrt{\sum_{i=1}^{N}\left(y_{i}-\bar{y}\right)^{2}}}\right)
$$

where for the $i$-th datapoint, $\widehat{y}_{i}$ is the model output in response to the measured input that leads to the real system output $y_{i}$ and, $\bar{y}$ is the average of $y$. This index can effectively be used also for cross-validation purposes by evaluating the model ability to capture data that are different from those used for the identification of its parameters.

\section{Results}

The results achieved in the estimation of model parameters across the different scenarios are described below, the validation results are summarised in Table 1:

- Model 1 - Scenarios I and II: $\left[d_{1}, v_{2}, d_{2}, b, x_{1}(0), x_{2}(0)\right]=$ [0.0047, 0.0078, 0.0124, 0.0035, 1.0617, 1.2211], (Supporting Figure 3, Panels A and B)

- Model 2 - Scenarios III and IV: $\left[d_{1}, v_{2}, d_{2}, b, x_{1}(0), x_{2}(0)\right]=$ [0.0063, 0.0274, 0.0166, 0.0018, 1.0343, 1.0424], (Supporting Figure 3, Panels C and D)

Table 1: Identification results

\begin{tabular}{|l|c|c|c|c|}
\hline & Scenario I & Scenario II & Scenario III & Scenario IV \\
\hline FPE & 0.0043 & 0.0148 & 0.0032 & 0.0124 \\
\hline AIC & -5.3900 & -4.2131 & -5.7500 & -4.3901 \\
\hline FIT (\%) & 67.18 & 40.88 & 78.84 & 58.26 \\
\hline
\end{tabular}

The two models are able to predict the experimental data across all the identification and validation scenarios (Supporting Figure 3 A-D). 
The two input signals (Supporting Figure 1 and 2, lower panels), used for the identification, have different frequencies (number of switches per time unit), the second higher than the first; this results in different dynamical properties for the two models obtained.

To explore these differences it is worth to recapitulate and use some basic concepts of System Theory: a) the step response of a system is the time behaviour of the output when the input changes from 0 to 1 in a very short time (step input), b) the time constant, denoted by $\tau$ is the parameter characterising the step response of a linear system; the smaller is the time constant, the faster is the response to input variations (3). In the case of state space linear systems, with order greater than one, the time constant $\tau$ can be calculated as the inverse of the smaller eigenvalue associated to the system itself. Specifically, assuming the model structure is:

$$
\dot{x(t)}=A x(t)+B u(t), \quad x(0)=x_{0}
$$

with the matrix $A$ being lower diagonal, i.e.:

$$
A=\left(\begin{array}{cc}
-d_{1} & 0 \\
v_{2} & -d_{2}
\end{array}\right)
$$

it is possible to demonstrate that the eigenvalues are equal to the elements on the diagonal, and for both the models the smaller eigenvalue is $d_{1}$. Thus model 1 has a time constant $\tau_{1}=212 \mathrm{~min}$ and model 2 has a time constant $\tau_{2}=159 \mathrm{~min}$. It is not surprising that model 2, identified using the input signal with the highest frequency, is the faster. This explains why, in the cross validations scenarios II and IV, the second model performs better than the first; model 2, being inferred starting from the high frequency input, is capable of responding promptly even to a slower signal, conversely model 1, for fast stimuli, behaves as a filter not reproducing properly system behaviour in response to high frequency signals. Furthermore, as it is possible to appreciate from Supporting Figure 2, the input signal (Lower Panel) and cell fluorescence (Upper Panel) are highly correlated, thus the most reliable model describing 
this system behaviour is model 2 .

From the results achieved in fitting the experimental data, we chose model 2 as the mathematical description of the process to perform in-silico control experiments.

\section{PI implementation and anti-windup compensation}

In practical control implementations the control signal $\hat{u}(t)$ is fed to the process being regulated by means of actuators (i.e. motors, valves, pumps). These components have physical limitations: motors have limited speed and acceleration, valves cannot be more than fully opened or fully closed and pumps cannot go slower than stopped. Thus the control signal acting on the system is saturated between the minimum and the maximum values achievable with the actuator being used.

If the control variable $\hat{u}$ exceeds the saturation limits, the actuator will constantly run at its saturation limits, thus the feedback loop becomes unresponsive to the error being measured and the error remains nonzero. In the case of PI or PID feedback control strategy, the error is integrated and the integral term may become very large, hence the control signal remains saturated even if the error changes and, it may take a long time before the integrator and the controller output return inside the saturation range. This situation is called integrator windup and leads to large transient in system response.

To fulfil the constraints on the control input, a modulation was implemented on PI's output (main text, Methods section). Specifically the modulation introduces a saturation on the regulator output thus leading to a possible integrator windup. To overcome this issue we introduced in our feedback control loop an anti-windup scheme proposed in (3) and shown in Supporting Figure 4. We also compared in-silico the performance of the PI with and without the anti-windup compensation in accomplishing the set-point regulation

task (Supporting Figure 5 A-B), and the signal tracking of the staircase control reference (Supporting Figure $5 \mathrm{C}$-D). We note that the system output reaches the control objective 
faster when being controlled with the anti-windup compensation (set-point control) and exhibits less oscillations around the reference (staircase control reference).

\section{MPC implementation}

To speed up the computation process during the minimisation of the sum of the squared control error (Equation 3, main text), we decided to use a discretised version of model 2 assuming that the input is piece-wise constant during the sampling period $T$ (zero-order hold method described in (4)), thus obtaining:

$$
x_{k+1}=A x_{k}+B u_{k}, \quad y_{k}=C x_{k} . \quad x(0)=x_{0}
$$

where $x_{k}=\left(\begin{array}{l}x_{1}(k T) \\ x_{2}(k T)\end{array}\right)$ is the system state, $u_{k}=u(k T)$ is the input and $y_{k}=y(k T)$ is the output, with $k$ being a natural number $(k \in[1,2, \ldots])$. Here the matrices $A, B$ and $C$ are:

$$
\begin{gathered}
A=\left(\begin{array}{cc}
a_{1,1} & 0 \\
a_{2,1} & a_{2,2}
\end{array}\right) \\
B=\left(\begin{array}{l}
b_{1} \\
0
\end{array}\right) \\
C=\left(\begin{array}{ll}
0 & c_{2}
\end{array}\right)
\end{gathered}
$$

The control input, as in the continuous time case, affects directly only the first system equation via the coefficient $b_{1}$, and it can take values $u_{M A X}=2$ and $u_{M I N}=0$. 


\section{ZAD implementation}

The ZAD controller, as the MPC, uses a mathematical description of the system being controlled to compute at each sampling time the control action. To carry out real time control experiment with this nonlinear feedback strategy we used the same model employed in the case of the MPC implementation.

Moreover the ZAD control technique, fulfils the sliding condition typical of the Sliding Mode Control only on average over each sampling period $k T$ (5), thus allowing to directly calculate the duty cycle $d_{k}$ via the solution of the integral equation:

$$
\mathbb{E}_{T}[s(x(t))]=\frac{1}{T} \int_{k T}^{(k+1) T} s(x(t)) d t=0
$$

The solution of (12) can be computationally expensive, thus to overcome this issue we considered the piecewise-linear approximation of the sliding surface $s(x(t))$ proposed in $(6)$

$$
s(x(t))= \begin{cases}s_{k}+(t-k T) \dot{s}_{k}^{\text {on }} & k T \leq t<\left(k+d_{k}\right) T \\ s_{k}+d_{k} T \dot{s}_{k}^{\text {on }}+\left(t-\left(k+d_{k}\right) T\right) \dot{s}_{k}^{\text {off }} & \left(k+d_{k}\right) T \leq t<(k+1) T\end{cases}
$$

where $s_{k}, \dot{s}_{k}^{\text {on }}$, and $\dot{s}_{k}^{\text {off }}$ are given by:

$$
\begin{gathered}
s_{k}=s\left(x_{k}\right) \\
\dot{s}_{k}^{\text {on }}=\left.\dot{s}\left(x_{k}\right)\right|_{u=2} \\
\dot{s}_{k}^{\text {off }}=\left.\dot{s}\left(x_{k}\right)\right|_{u=0}
\end{gathered}
$$

All the following solution steps have been already described in ( 7$)$, we adapted them to our specific case where the sliding surface is defined as:

$$
s(x(t))=\left(x_{2}(t)-x_{2_{r e f}}(t)\right)+\left(\dot{x}_{2}(t)-\dot{x}_{2_{r e f}}(t)\right)
$$


By substituting the piecewise-linear approximation (13) into (12) we obtained:

$$
\begin{aligned}
\mathbb{E}_{T}[s(x(t))]= & \frac{1}{T} \int_{k T}^{\left(k+d_{k}\right) T}\left[s_{k}+(t-k T) \dot{s}_{k}^{\text {on }}\right] d t \\
& +\frac{1}{T} \int_{\left(k+d_{k}\right) T}^{(k+1) T}\left[s_{k}+d_{k} T \dot{s}_{k}^{\text {on }}+\left(t-\left(k+d_{k}\right) T\right) \dot{s}_{k}^{\text {off }}\right] d t
\end{aligned}
$$

and by computing the integral in(16):

$$
\mathbb{E}_{T}[s(x(t))]=0 \Longrightarrow \frac{1}{2} d_{k}^{2} T\left(\dot{s}_{k}^{\text {off }}-\dot{s}_{k}^{\text {on }}\right)-d_{k} T\left(\dot{s}_{k}^{\text {off }}-\dot{s}_{k}^{\text {on }}\right)+s_{k}+\frac{1}{2} T \dot{s}_{k}^{\text {off }}=0
$$

The duty cycle $d_{k}$ can then be calculated by solving the quadratic equation (17), thus obtaining:

$$
d_{k}=\frac{-T\left(\dot{s}_{k}^{\text {on }}-\dot{s}_{k}^{\text {off }}\right) \pm \sqrt{T\left(\dot{s}_{k}^{\text {on }}-\dot{s}_{k}^{\text {off }}\right)\left(2 s_{k}+T \dot{s}_{k}^{\text {on }}\right)}}{-T\left(\dot{s}_{k}^{\text {on }}-\dot{s}_{k}^{\text {off }}\right)}
$$

Moreover, considering that:

$$
\dot{s}_{k}^{\text {off }}-\dot{s}_{k}^{\text {on }}=-2 b_{1} a_{2,1}<0 \quad \Longrightarrow \quad \dot{s}_{k}^{\text {on }}-\dot{s}_{k}^{\text {off }}>0
$$

the solutions of (12) are:

$$
d_{k}=1 \mp \sqrt{\frac{2 s_{k}+T \dot{s}_{k}^{\text {on }}}{T\left(\dot{s}_{k}^{\text {on }}-\dot{s}_{k}^{\text {off }}\right)}}
$$

As the duty cycle assumes values only in $[0,1]$, the only admissible solution is:

$$
d_{k}=1-\sqrt{\frac{2 s_{k}+T \dot{s}_{k}^{\text {on }}}{T\left(\dot{s}_{k}^{\text {on }}-\dot{s}_{k}^{\text {off }}\right)}}
$$

Furthermore, to avoid saturation, we imposed the constraint:

$$
0 \leq \frac{2 s_{k}+T \dot{s}_{k}^{\text {on }}}{T\left(\dot{s}_{k}^{\text {on }}-\dot{s}_{k}^{\text {off }}\right)} \leq 1
$$




\section{Control algorithm implementations}

To perform in-vivo control experiments, we integrated the implementation of the PI, MPC and ZAD controllers into the experimental platform. At the beginning of each experiment cells are loaded into the microfluidic device, and are fed for 180 min with Galactose. This was done for two main reasons: a) to allow them to adapt to the microfluidic environment and b) to calculate the high steady - state of fluorescence since the reference signal is expressed as a percentage of this value (calibration phase). To integrate the control strategies into the experimental platform, we implemented them as a Finite State Automaton (FSA) in MATLAB programming environment. The FSA works as follows: after the calibration phase, at each control step $(k)$ an image is acquired by the microscope, and the normalised fluorescence signal is computed thanks to the image processing algorithm described in $(8,9)$. The fluorescence signal $y(k)$ is compared against the reference signal $r(k)$, to obtain the error $e(k)$. The control input $u(k)$ is then computed accordingly to the control strategy adopted, hence the duty-cycle $d_{k}$ of the input over the sampling time is calculated. At the next instant $(k+1)$ a new image is acquired and the feedback computation takes place. The error $e(k+1)$ is available for a new control iteration and each step is repeated.

\section{Performance indices}

To assess and compare control performance from the three control algorithms, we used metrics based on the analysis of the control error $e$. These indexes in general are adopted to optimise the tuning of PI and PID regulator gains on the basis of the control outcome (3), hence they provide a valid measure of control quality. The Integral Square Error (ISE), defined as:

$$
I S E=\int_{0}^{t} e(\tau)^{2} d \tau
$$

integrates the square of the error over the time. ISE penalises large errors more than smaller ones. 
The Integral Absolute Error (IAE), given by:

$$
I A E=\int_{0}^{t}|e(\tau)| d \tau
$$

integrates the absolute error of the control over time; a weighted version of the IAE is the Integral Time Absolute Error (ITAE) calculated as:

$$
I T A E=\int_{0}^{t} \tau|e(\tau)| d \tau
$$

that integrates the absolute error multiplied by the time. It penalises more persisting errors than those at the start of the response.

\section{Experimental Protocol}

\section{Cells and microfluidic device set up}

On day 0 batch cultures are inoculated in $10 \mathrm{~mL}$ GAL/RAF+Sulforhodamine B (SigmaAldrich) (2\%) Synthetic Complete medium (SC). On day 1 the batch culture is diluted at intervals of 12 hours (final $\mathrm{OD}_{600}$ 0.01). On day 2, 60mL syringes (Becton, Dickinson and Company, NJ) filled with $10 \mathrm{~mL} \mathrm{SC}+\mathrm{GAL} / \mathrm{RAF}(2 \%)$ and $\mathrm{SC}+\mathrm{GLC}(2 \%)$ media are prepared, as well as sink syringes (filled with $10 \mathrm{~mL}$ ddH2O); capillaries and needles are used to allow connection to the microfluidic device. Temperature in the micro-environment surrounding the moving stage of the microscope is allowed to settle at $30{ }^{\circ} \mathrm{C}$. Before connecting media and sink syringes, the microfluidic device MFD0005a wetting is carried out as described in (10). After air bubbles are removed, media and water filled $60 \mathrm{~mL}$ syringes are attached to the device and correct functioning is checked by inspecting the red-fluorescence emitted by Sulforhodamine B as a result of the automatic height control of syringes. This allowed us to carry out a correct calibration of the actuation strategy before the actual experiment is run. At this point cells (yGIL337 strain) are injected in the microfluidic device 
by pouring the batch culture in a $60 \mathrm{~mL}$ syringe similar to the ones used to media and sinks. Once cells are trapped in the defined area (see (10) for details) Perfect Focus System is activated to assist autofocusing during the experiment and the acquisition routine of the microscope software is started to initiate image acquisition.

\section{Calibration phase}

The calibration phase, during which cells are kept in Galactose, is needed both to let cells adapt to the microfluidic environment, and to set the unit of measure of fluorescence, which may vary due to technical and biological variability in each experiment.

Once cells are loaded in the microfluidic device, they are kept in a Galactose enriched growing medium for 180 minutes by controlling the actuators in order to hold the syringe filled with Galactose in a higher position with respect to the one carrying Glucose.

\section{GAL1 promoter identification experiments}

After the calibration phase of the experiment a sequential MATLAB script controls syringes position over the time to obtain desired time profile for the input fed to cells. The image processing algorithm, running in real time, calculates the absolute fluorescence emitted by the entire cell population as well as its normalised value by dividing the time course of fluorescence by the average fluorescence intensity measured during the initial calibration phase. Input and output time series thus generated are used to apply System Identification techniques.

\section{$G A L 1$ promoter control experiments}

The following experimental procedure applies to control experiments carried out on cells of the yGIL337 strains used in this study. 
Set - point control experiments: once cells are loaded in the microfluidic device, the user has to start a custom MATLAB script, that manages the entire experimental platform (controller implementation, actuation, image analysis), and has to set the duration (in minutes) of the control. The script is built to calculate the set point for the control as a percentage (indicated by the user at the beginning of the experiment) of the average of the fluorescence measured by the image processing algorithm during the calibration phase previously described. After this the implemented script proceeds in executing all the code blocks necessary to reach and maintain the fluorescence reference.

Signal - tracking control experiments: the length and the values of the steps of the staircase reference used in signal tracking control experiments with GAL1 promoter cells is calculated by a custom MATLAB script that manages the entire experimental platform. The script is built to calculate the values of each of the steps as percentages (indicated by the user at the beginning of the experiment in the case of the staircase control reference) of the average of the fluorescence measured by the image processing algorithm during the calibration phase. At the end of the calibration, the implemented script proceeds in executing all the code blocks necessary to reach and maintain the fluorescence reference. The same procedure, with a different calculation for the control reference, applies to the case of signal tracking control with the ramp reference signal and the sinusoidal wave. 


\section{References}

1. Fiore, G., Menolascina, F., di Bernardo, M., and di Bernardo, D. (2013) An experimental approach to identify dynamical models of transcriptional regulation in living cells. Chaos: An Interdisciplinary Journal of Nonlinear Science 23, 025106-025106.

2. Ljung, L. System identification; Wiley Online Library, 1999.

3. Aström, K. J., and Murray, R. M. Feedback systems: an introduction for scientists and engineers; Princeton University Press, 2010.

4. Franklin, G. F., Powell, J. D., and Workman, M. L. Digital control of dynamic systems; Addison-wesley Menlo Park, 1998; Vol. 3.

5. Fossas, E., Grinó, R., and Biel, D. (2001) Quasi-Sliding control based on pulse width modulation, zero averaged dynamics and the L2 norm. Advances in Variable Structure System, Analysis, Integration and Applications 335-344.

6. Ramos, R. R., Biel, D., Fossas, E., and Guinjoan, F. (2003) A fixed-frequency quasisliding control algorithm: application to power inverters design by means of FPGA implementation. Power Electronics, IEEE Transactions on 18, 344-355.

7. D'Amico, M. B., and Angulo, F. (2013) Performance of a Zero Average Dynamicscontrolled buck converter using different pulse-width modulation schemes. International Journal of Circuit Theory and Applications

8. Menolascina, F., Fiore, G., Orabona, E., De Stefano, L., Ferry, M., Hasty, J., di Bernardo, M., and di Bernardo, D. (2014) In-Vivo Real-Time Control of Protein Expression from Endogenous and Synthetic Gene Networks. PLoS Computational Biology 10, e1003625.

9. La Brocca, R., Menolascina, F., di Bernardo, D., and Sansone, C. (2011) Segmentation, tracking and lineage analysis of yeast cells in bright field microscopy images. Interna- 
tional Workshop on Pattern Recognition in Proteomics, Structural Biology and Bioinformatics - Ravenna, Italy

10. Ferry, M., Razinkov, I., and Hasty, J. (2011) Microfluidics for synthetic biology from design to execution. Methods Enzymol 497, 295. 


\section{Supporting Figures}
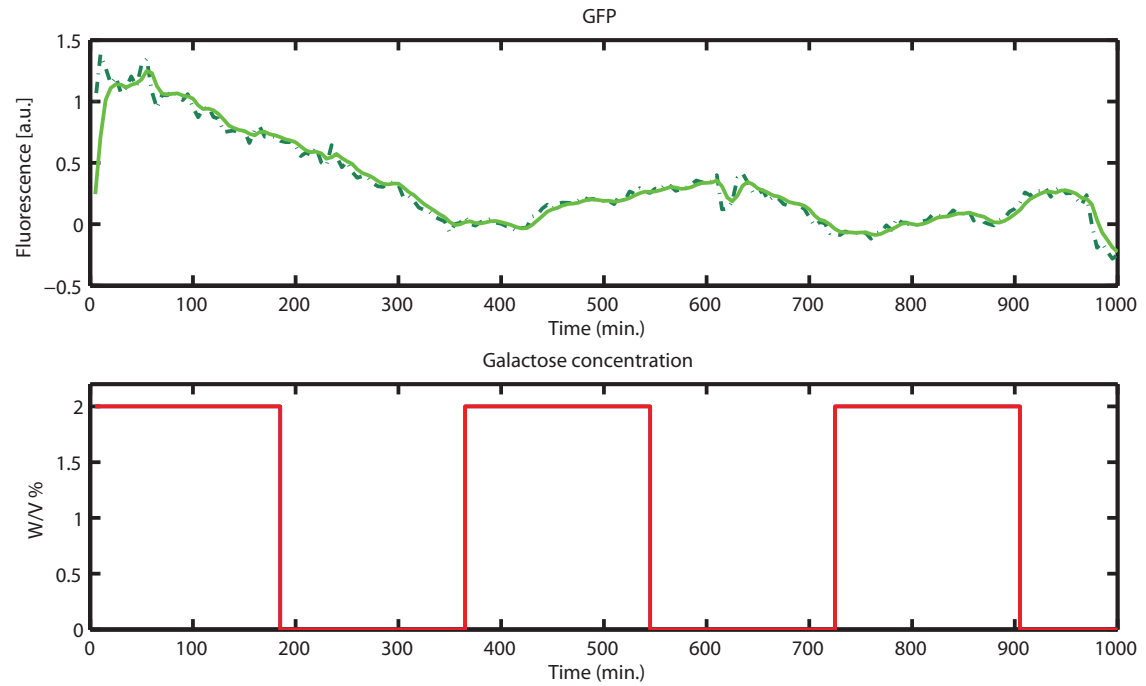

Figure 1: Experimental data set 1. Upper Panel: Cell population average fluorescence (dashed green line) measured during the experiment. Lower Panel: Input signal, the high level corresponds to Galactose, whereas the low one corresponds to Glucose. 

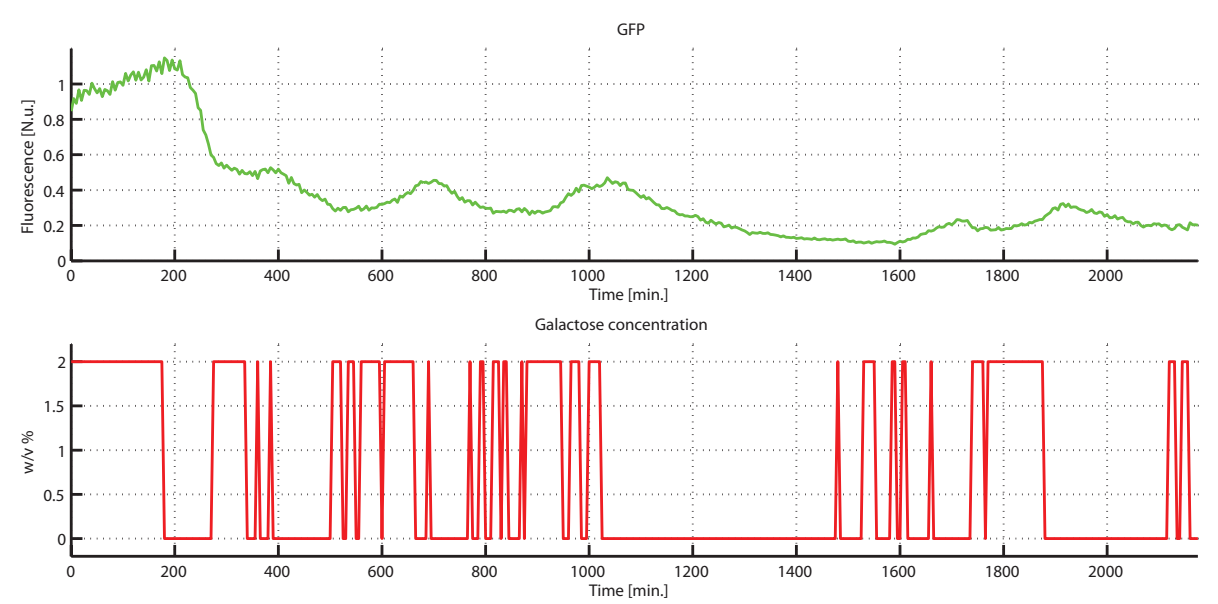

Figure 2: Experimental data set 2. Upper Panel: Cell population average fluorescence (green line) measured during the experiment. Lower Panel: Input signal, a high level corresponds to Galactose, whereas a low level to Glucose, after the first 180 minutes in which cells are fed with Galactose, the input is calculated as a random sequence of pulses in between Galactose and Glucose.
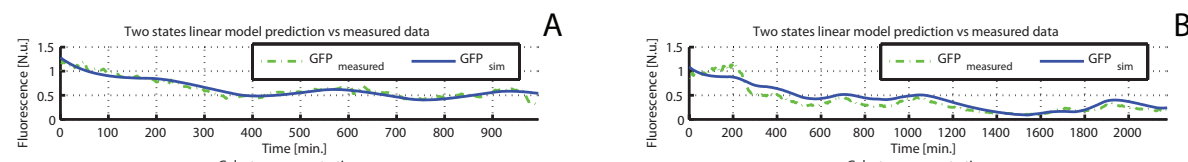

B
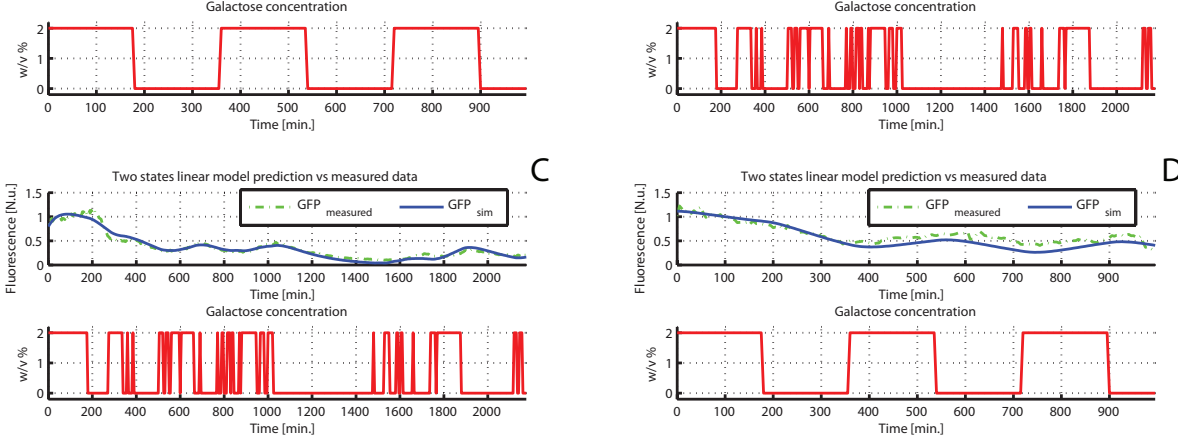

Figure 3: Identification and validation results of two state space linear models. (A) The solid blu line represents the output of the inferred model in response to the input (red square wave) used for the identification, the dashed green line is the measured cell fluorescence. (B) The model inferred with experimental data of (A), has been validated in reproducing the measured cell fluorescence (dashed green line) obtained in response to the selected input (red square wave); the result of model simulation, carried out with the same input, is represented by the solid blue line $(\mathrm{C})$ The solid blu line represents the output of the inferred model in response to the input (red square wave) used for the identification, the dashed green line is the measured cell fluorescence. (D) The model inferred with experimental data of $(C)$, has been validated in reproducing the measured cell fluorescence (dashed green line) obtained in response to the selected input (red square wave); the result of model simulation, carried out with the same input, is represented by the solid blue line 


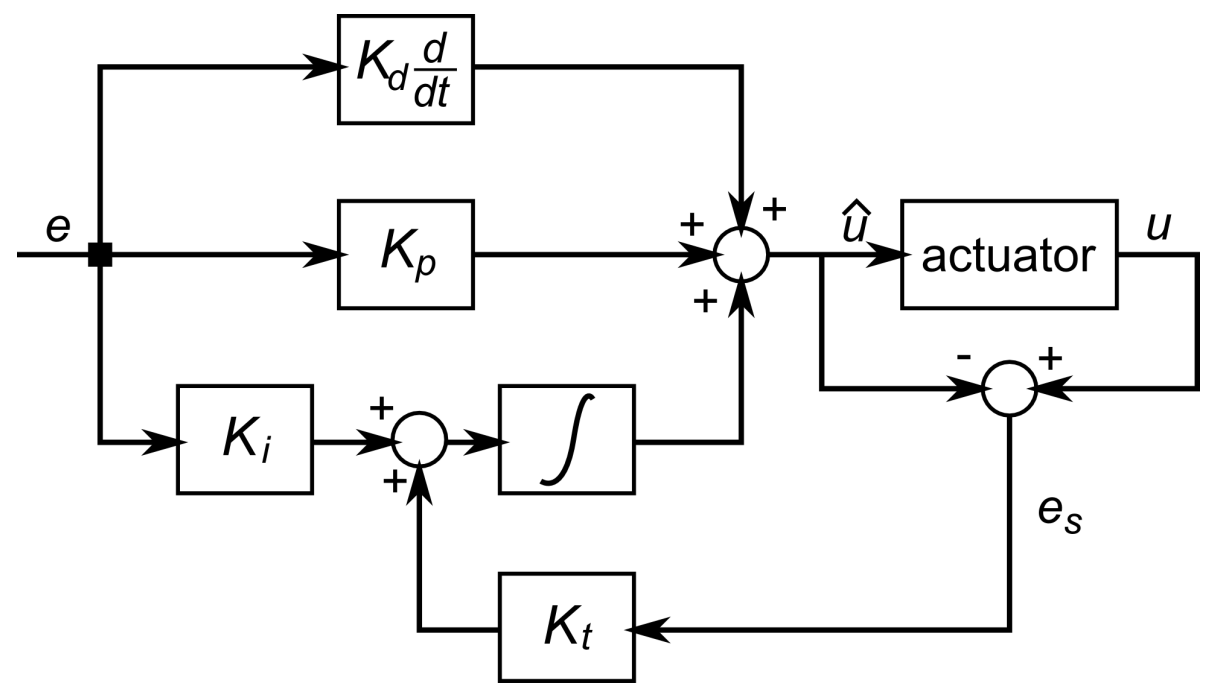

Figure 4: PID controller with anti-windup. The input to the integrator consists of the error term plus a "reset" based on input saturation. If the actuator is not saturated $e_{s}=u-\hat{u}=0$, otherwise $e_{s}$ will decrease the integrator input to prevent windup.
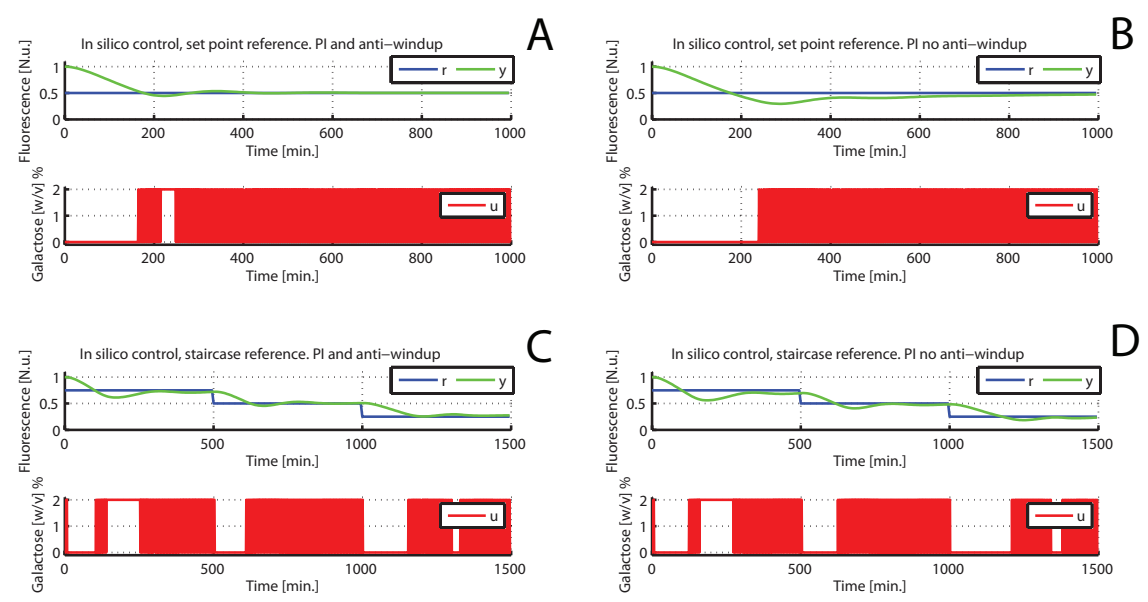

Figure 5: In silico control with and without windup compensation. The blue line is the reference signal $(r)$. The green line is the simulated fluorescence level $(y)$. The red line is the control input $(u)$. (A-D) Four in-silico control experiments performed on the GAL1 promoter mathematical model by the means of the PI control algorithm in the case of setpoint control with (A) and without anti-windup compensation (B) and, to accomplish the tracking of the staircase reference signal with $(\mathrm{C})$ and without anti-windup compensation (D). 